\title{
Design of Hybrid Electrochromic Materials with Large Electrical Modulation of Plasmonic Resonances
}

Petr A. Ledin, ${ }^{a}$ Ju-Won Jeon, ${ }^{a}$ Jeffrey A. Geldmeier, ${ }^{a}$ James F. Ponder Jr., ${ }^{b}$ Mahmoud A. Mahmoud, ${ }^{c}$ Mostafa El-Sayed, ${ }^{c}$ John R. Reynolds, ${ }^{b}$ Vladimir V. Tsukruk ${ }^{* a}$

a School of Materials Science and Engineering, Georgia Institute of Technology, Atlanta, Georgia 30332-0245 (USA)

${ }^{b}$ School of Chemistry and Biochemistry, School of Materials Science and Engineering, Center for Organic Photonics and Electronics, Georgia Tech Polymer Network, Georgia Institute of Technology, Atlanta, Georgia 30332-0400 (USA)

${ }^{\mathrm{C}}$ Laser Dynamics Laboratory, School of Chemistry and Biochemistry, Georgia Institute of Technology, Atlanta, Georgia 30332-0400 (USA)

${ }^{*}$ Corresponding e-mail: vladimir@mse.gatech.edu 


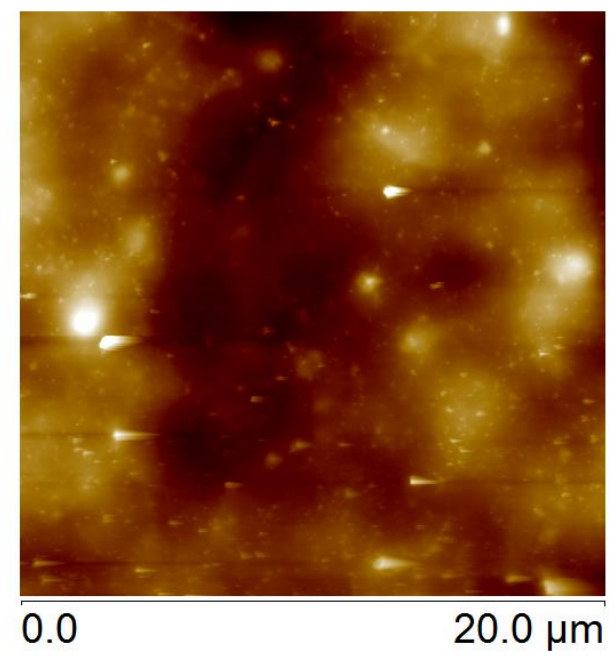

$150.0 \mathrm{~nm}$

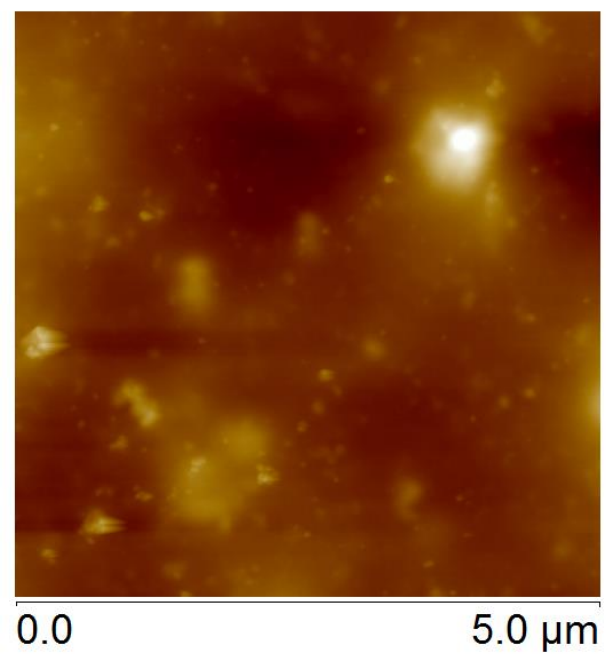

$100.0 \mathrm{~nm}$

$-100.0 \mathrm{~nm}$

Figure S1. Topographical AFM images of spraycoated ECP-Magenta films. 


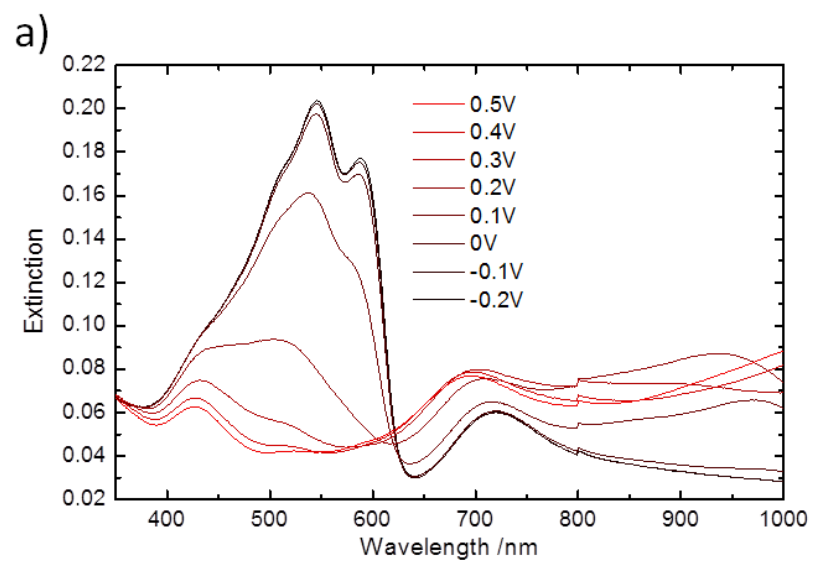

b)

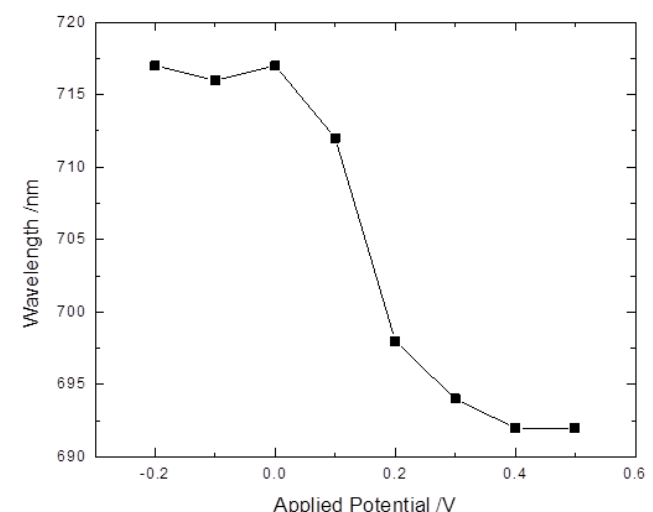

c)

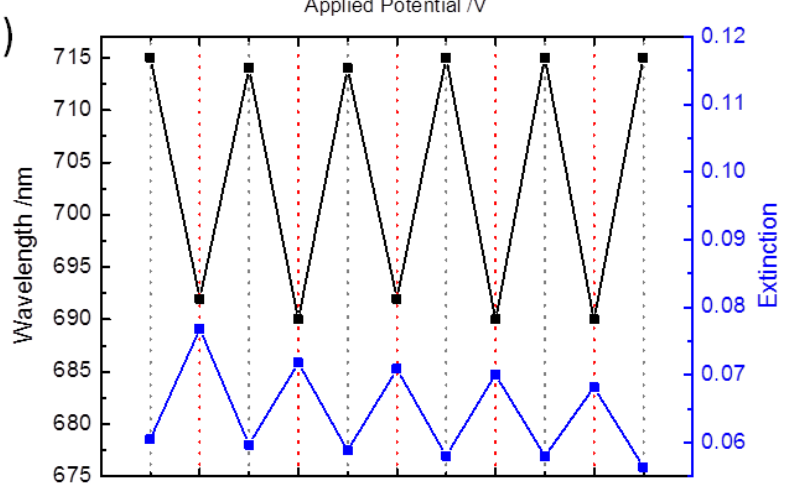

Figure S2. Electrochromic behavior of AuNR/ECP-Mg composite material with $60 \mathrm{~nm}$ polymer thickness under applied potential. a) Change in extinction over one cycle. b) LSPR peak wavelength plotted versus applied potential. c) Electrochemical cycling for 5 cycles. Red dotted lines indicate electrochemical oxidation. 

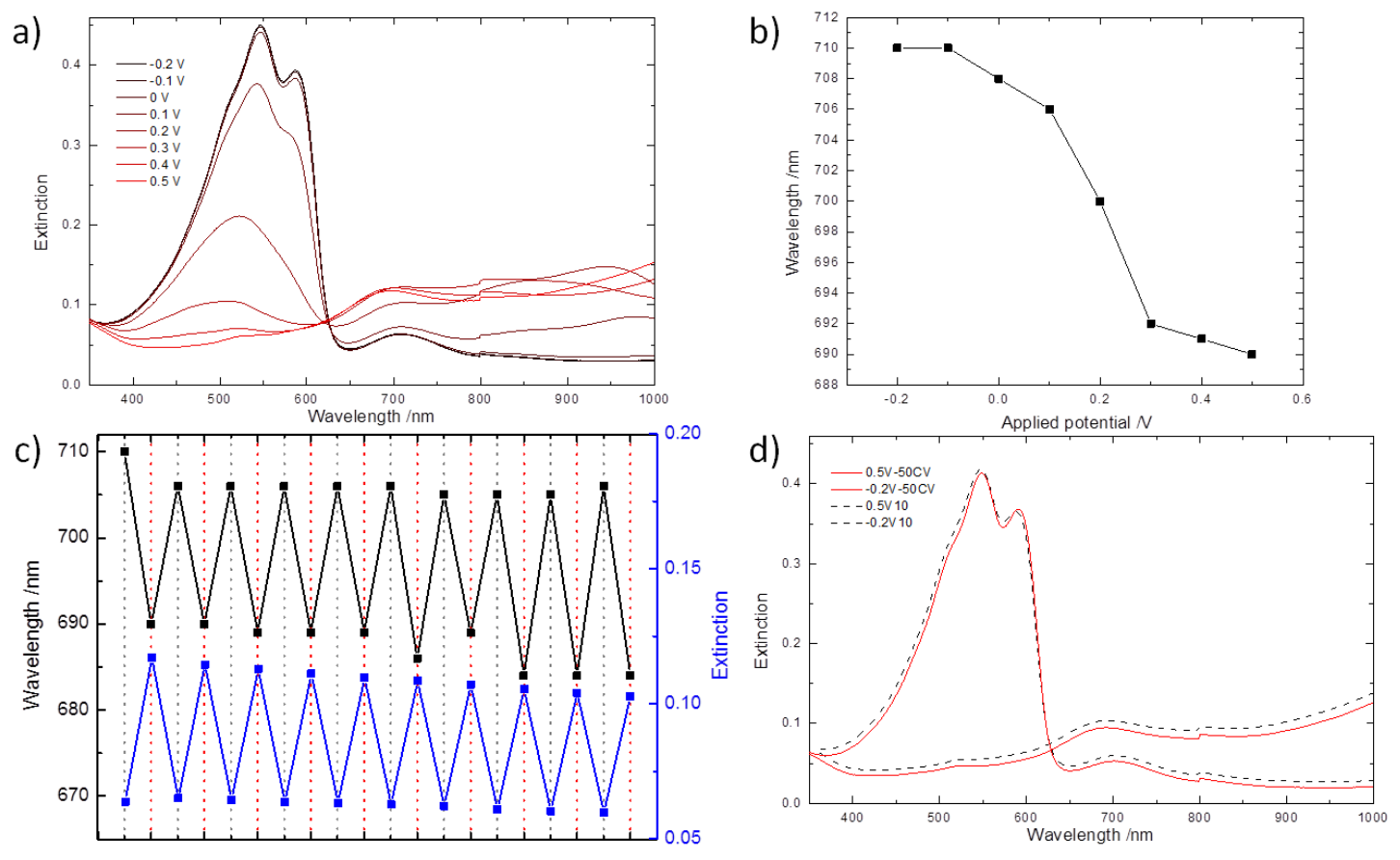

Figure S3. Electrochromic behavior of AuNR/ECP-Mg composite material with $120 \mathrm{~nm}$ polymer thickness under applied potential. a) Change in extinction over one cycle. b) LSPR peak wavelength plotted versus applied potential. c) Electrochemical cycling for 10 cycles. Red dotted lines indicate electrochemical oxidation. d) Stability before and after $50 \mathrm{CV}$ cycles. 


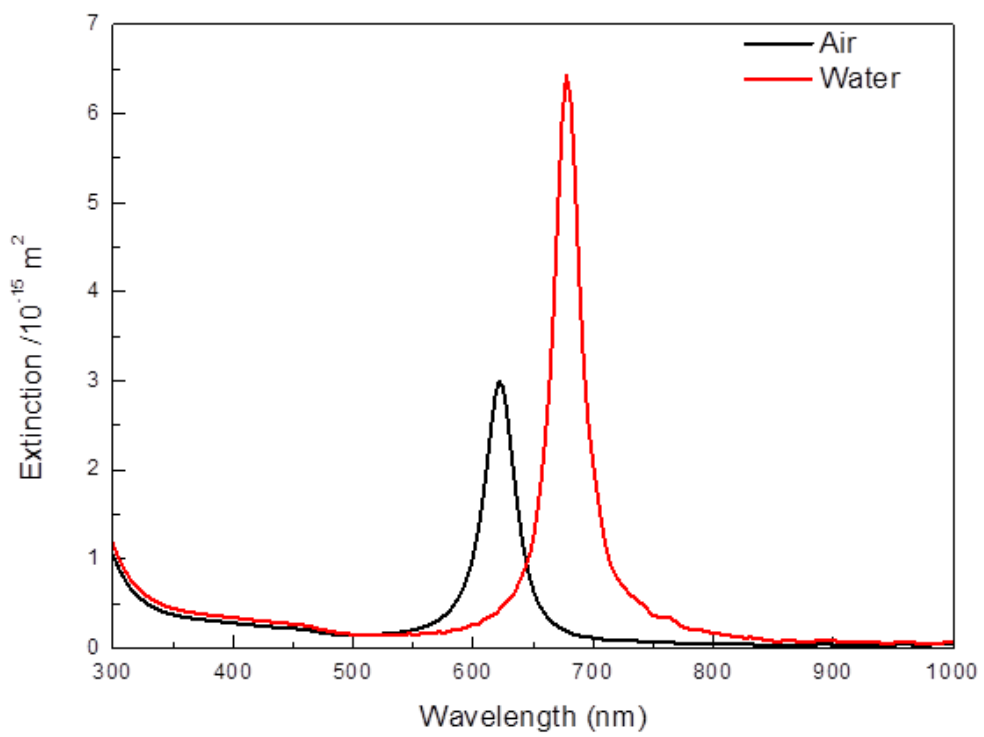

Figure S4. Simulated extinction spectra of AuNRs on ITO substrate in air and water.

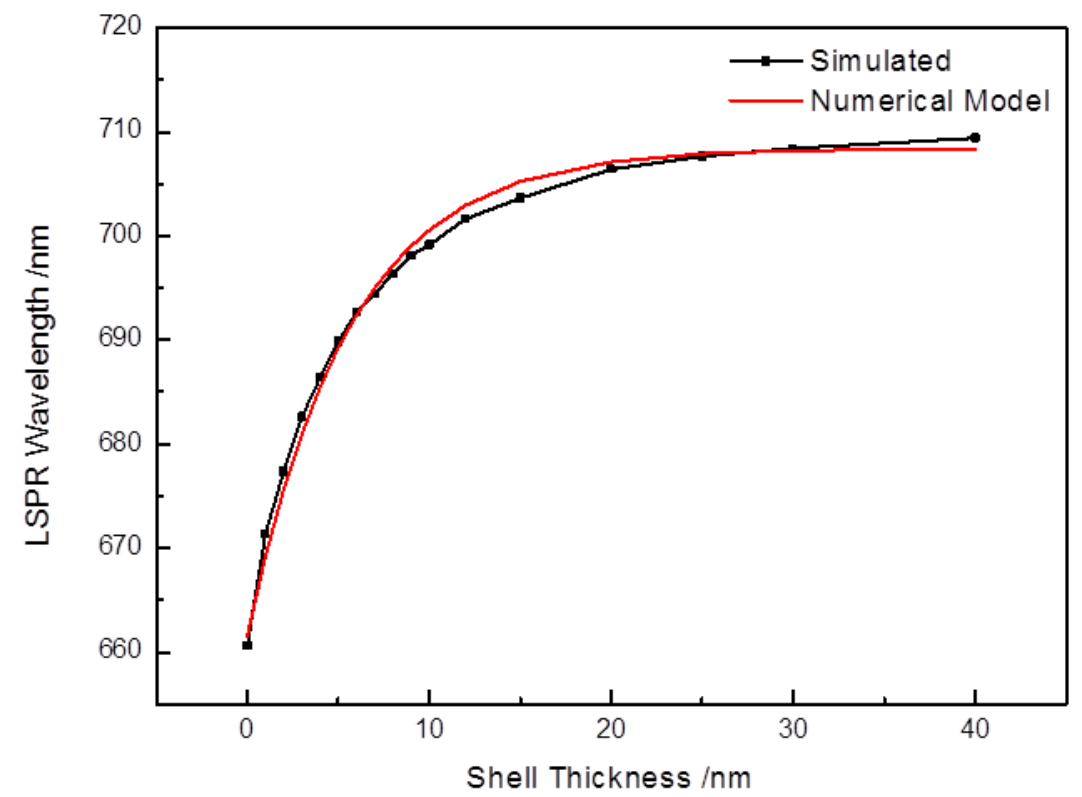

Figure S5. Simulated LSPR wavelength of gold nanorods with various silica shell thicknesses (black line). Exponential fit to equation 1 to determine the electromagnetic field decay length $\left(I_{d}\right)$ (red line). 